\title{
Stability and Bifurcation Analysis of Functionally Graded Materials Plate Under Different Loads and Boundary Conditions
}

\author{
Shuping Chen ${ }^{1, a}$, Wei Zhang ${ }^{2}$ and Yuxin $\mathrm{Hao}^{3}$ \\ 1 College of Mathematics, Xiamen University of Technology, Xiamen 361024, P. R. China \\ ${ }^{2}$ College of Mechanical Engineering, Beijing University of Technology, Beijing 100124, P. R. China \\ ${ }^{3}$ College of Mechanical Engineering, Beijing Information Science and Technology University, Beijing 100192, P. R. \\ China
}

\begin{abstract}
This paper presents the stability and bifurcation analysis of a simply-supported functionally graded materials (FGMs) rectangular plate subject to the transversal and in-plane excitations. A two-degree-of-freedom nonlinear system of the FGM plate is obtained via the Hamilton's principle and the Galerkin approach. The case of primary parametric resonance and 1:2 internal resonance is considered. The asymptotic perturbation method is utilized to obtain four-dimensional nonlinear averaged equation. With the aid of Matlab and normal form theory, the various types of dynamical behavior in the neighborhood of a kind of degenerated equilibrium point are investigated. It was found that static bifurcation and Hopf bifurcation exist for the FGM rectangular plate under certain conditions.
\end{abstract}

\section{Introduction}

The functionally graded materials (FGMs) are a kind of microscopically composite materials. Their properties can be spatially varied to achieve the desired structural, electrical functions, or thermal. It is emerging with great potential in mechanical science research because of their high performance and multi-function [1-3].

For FGM plate, many studies have concentrated on the nonlinear dynamic behavior analysis. Sladek et al. [4] applied the meshless local Petrov-Galerkin method to a circular plate with functionally graded piezoelectric material properties. Li et al. [5] studied the free vibration of functionally graded material sandwich rectangular plates with simply supported and clamped edges based on the three-dimensional linear theory of elasticity. Allahverdizadeh et al. [6] developed a semianalytical approach for nonlinear free and forced axisymmetric vibration of a thin circular functionally graded plate. Gunes and Reddy [7] investigated the geometrically nonlinear analysis of functionally graded circular plates subjected to mechanical and thermal loads. Hao [8] investigated the nonlinear dynamics of a simply-supported FGM rectangular plate with the parametric and forcing excitations.

Many studies have considered the stability and bifurcation of nonlinear systems. Zhang [9] investigated the stability and bifurcation behaviors of a two-dimensional nonlinear viscoelastic panel in supersonic flow by using analytical and numerical methods. Anton et al. [10] applied the stochastic

\footnotetext{
${ }^{\mathrm{a}}$ Corresponding author : shupingchen@126.com
} 
normal form to an aeroelastic random dynamical system with uncertainties in the bifurcation parameter and the plunge and pitch nonlinear terms. Li et al. [11] analyzed the stabilities and bifurcations of a cantilevered plate with nonlinear motion constraints in an axial subsonic flow.

This paper focuses on research on the bifurcation and stability analysis of the FGM rectangular plate deflection with variation of material properties. Based on the theoretical model for the nonlinear oscillations of the FGM plate subjected to the transversal and in-plane excitations, the normal form theory and bifurcation theory are applied to discuss the local dynamical behaviors of the FGMs rectangular plate.

\section{The functionally graded materials plate model}

In this section, we use the normal form method to the rectangular plate made of FGMs with length $a$, width $b$ and thickness $h$ as presented in Fig.1. The plane is subjected to in-plane and transverse excitations. A Cartesian coordinate system is located in the middle surface of the FGMs rectangular plate. $u, v$ and $w$ are the displacements of an point within the plate in the $x, y$ and $z$ directions. $-\left(p_{0}-p_{1} \cos \left(\Omega_{2} t\right)\right)$ represents the in-plane excitation along the width. The transverse excitation is written as $F(x, y) \cos \left(\Omega_{1} t\right)$. In this paper, the governing equations of transverse motion for the FGMs plate in dimensionless form can be expressed as follows: $[8,12,13]$

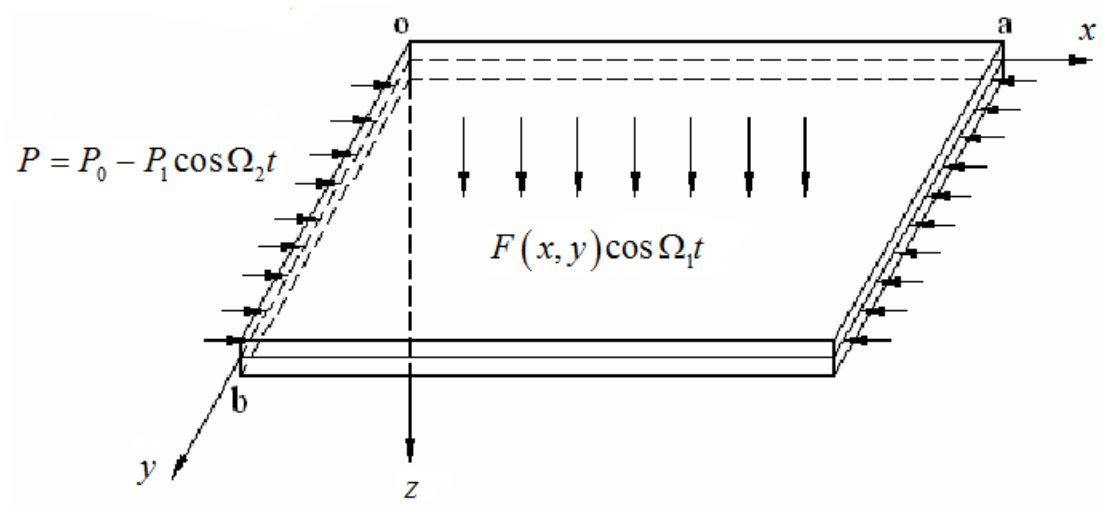

Figure 1. The model of a functionally graded materials rectangular plate

$$
\begin{aligned}
& \ddot{w}_{1}+\omega_{1}^{2} w_{1}+a_{1} \dot{w}_{1}+a_{2} w_{1} \cos \Omega_{2} t+a_{3} w_{1}^{2}+a_{4} w_{2}^{2}+a_{5} w_{1} w_{2}^{2}+a_{6} w_{1}^{3}+a_{7} w_{1} w_{2}=f_{1} \cos \Omega_{1} t, \\
& \ddot{w}_{2}+\omega_{2}^{2} w_{2}+b_{1} \dot{w}_{2}+b_{2} w_{2} \cos \Omega_{2} t+b_{3} w_{1} w_{2}+b_{4} w_{1}^{2}+b_{5} w_{2}^{2}+b_{6} w_{2} w_{1}^{2}+b_{7} w_{2}^{3}=f_{2} \cos \Omega_{1} t,
\end{aligned}
$$

where $a_{i}, b_{i}(i=1, \cdots, 7)$ are non-dimensional coefficients and can be found in the Reference [13].

In this paper, we investigate the case of 1:2 internal resonance and primary parametric resonance for the FGMs rectangular plate,

$$
\omega_{1}=\frac{\Omega_{1}}{2}+\varepsilon^{2} \sigma_{1}, \omega_{2}=\Omega_{1}+\varepsilon^{2} \sigma_{2}, \Omega_{1}=\Omega_{2}=\Omega,
$$

where $\sigma_{1}$ and $\sigma_{2}$ are two detuning parameters.

Using the Galerkin method and the asymptotic perturbation method, the four-dimensional averaged equation of a FGMs plate is defined by

$$
\begin{aligned}
\dot{x}_{1}= & -\mu_{1} x_{1}+\left(\sigma_{1}+\alpha_{1}+\alpha_{2} f_{1}\right) x_{2}+14 \alpha_{2} x_{1}^{2} x_{2}+14 \alpha_{2} x_{2}^{3}+\alpha_{3} x_{1} x_{2}^{2}+\alpha_{3} x_{1}^{3} \\
& +\alpha_{4} x_{2} x_{3}^{2}+\alpha_{4} x_{2} x_{4}^{2}+\alpha_{5} x_{2} x_{3}-\alpha_{5} x_{1} x_{4},
\end{aligned}
$$




$$
\begin{aligned}
\dot{x}_{2}= & \left(-\sigma_{1}+\alpha_{1}+\alpha_{2} f_{1}\right) x_{1}-\mu_{1} x_{2}-14 \alpha_{2} x_{1}^{3}-14 \alpha_{2} x_{2}^{2} x_{1}-\alpha_{4} x_{1} x_{3}^{2}-\alpha_{4} x_{1} x_{4}^{2} \\
& +\alpha_{3} x_{1}^{2} x_{2}+\alpha_{3} x_{2}^{3}+\alpha_{5} x_{1} x_{3}+\alpha_{5} x_{2} x_{4} \\
\dot{x}_{3}= & \beta_{1} f_{1} x_{2}-\mu_{2} x_{3}+\sigma_{2} x_{4}+\beta_{2} x_{1}^{2} x_{4}+\beta_{2} x_{2}^{2} x_{4}+\beta_{3} x_{4}^{3}+\beta_{3} x_{4} x_{3}^{2}+\beta_{4} x_{3}^{2} x_{2} \\
& +\beta_{4} x_{4}^{2} x_{2}-\beta_{5} x_{2}^{3}-\beta_{5} x_{1}^{2} x_{2}, \\
\dot{x}_{4}= & \beta_{1} f_{1} x_{1}-\sigma_{2} x_{3}-\mu_{2} x_{4}-\beta_{2} x_{1}^{2} x_{3}-\beta_{2} x_{2}^{2} x_{3}-\beta_{3} x_{3}^{3}+\beta_{3} x_{3} x_{4}^{2}+\beta_{5} x_{1}^{3} \\
& -\beta_{5} x_{2}^{2} x_{1}-\beta_{4} x_{3}^{2} x_{1}-\beta_{4} x_{4}^{2} x_{1}+\frac{1}{4} f_{2} .
\end{aligned}
$$

\section{Stability and bifurcation analysis of the FGMs plat}

In this section, the normal form theory and bifurcation theory is utilized to analyze the dynamical behaviors of the FGMs rectangular plate. The expression for the characteristic polynomial of equation (3) can be given as

$$
f(\lambda)=\lambda^{4}+c_{1} \lambda^{3}+c_{2} \lambda^{2}+c_{3} \lambda+c_{4},
$$

where

$$
\begin{aligned}
& c_{1}=2\left(\mu_{1}+\mu_{2}\right), \quad c_{2}=\sigma_{1}^{2}+\sigma_{2}^{2}+\mu_{1}^{2}+\mu_{2}^{2}+4 \mu_{1} \mu_{2}-\alpha^{2}, \\
& c_{3}=2 \mu_{1} \mu_{2}^{2}+2 \mu_{2} \mu_{1}^{2}+2 \mu_{1} \sigma_{2}^{2}+2 \mu_{2} \sigma_{1}^{2}-2 \mu_{2} \alpha^{2}, \\
& c_{4}=\mu_{1}^{2} \mu_{2}^{2}+\mu_{1}^{2} \sigma_{2}^{2}+\mu_{2}^{2} \sigma_{1}^{2}+\sigma_{1}^{2} \sigma_{2}^{2}-\mu_{2}^{2} \alpha^{2}-\sigma_{2}^{2} \alpha^{2},
\end{aligned}
$$

when $\alpha=\alpha_{1}+\alpha_{2} f_{1}, \gamma_{1}=\beta_{1} f_{1}$.

Let

$\mu_{1}=1, \mu_{2}=\sigma_{1}=0, \sigma_{2}=1, \alpha=1, \gamma_{1}=1, \alpha_{2}=\alpha_{3}=\alpha_{4}=\alpha_{5}=1, \beta_{2}=\beta_{3}=\beta_{4}=\beta_{5}=1$,

Therefore, the eigenvalues can be given by

$$
\lambda_{1}=0, \lambda_{2,3}= \pm i, \lambda_{4}=-2 \text {. }
$$

Letting $\mu_{1}=1+\varsigma_{1}, \mu_{2}=\varsigma_{2}$, and using the normal form theory and the cylinder coordinate transformation

$$
z_{1}=y, z_{2}=r \cos \theta, z_{3}=r \sin \theta, z_{4}=z_{4},
$$

The equations (3) can be rewritten as

$$
\begin{gathered}
\dot{y}=y\left(-\varsigma_{1}-\frac{12}{5} r^{2}-2 y^{2}\right), \\
\dot{r}=r\left(-\varsigma_{2}-\frac{119}{100} r^{2}-\frac{3}{10} y^{2}\right) .
\end{gathered}
$$

We can give some analyses to observe the dynamical behaviors of system (9).

(1) The original equilibrium solution

$$
y=r=0 \text {. }
$$

From equation (9), it is easy to see that the original equilibrium solution is stable when $\varsigma_{1}>0$ and $\varsigma_{2}>0$. Therefore, the invariant lines which represent the stability boundaries of the original equilibrium solution are $L_{1}: \varsigma_{2}=0$ and $L_{2}: \varsigma_{1}=0$.

(2) The static bifurcation solution

$$
y^{2}=-\frac{\varsigma_{1}}{2}, r=0 .
$$

It is obvious that the static bifurcation solution from the initial equilibrium solution takes place when $\varsigma_{1}<0$. It is to be noted that system (9) undergoes a static bifurcation on the curve: 


$$
T=\left\{\left(\varsigma_{1}, \varsigma_{2}\right): \varsigma_{1}<0,-\varsigma_{2}+\frac{3}{20} \varsigma_{1}<0\right\} .
$$

(3) The incipient Hopf bifurcation solution

$$
y=0, r^{2}=-\frac{100}{119} \varsigma_{2} .
$$

A family of limit cycles are bifurcated when $\varsigma_{2}<0$. We note that the incipient Hopf bifurcation solution is stable whenever $\varsigma_{2}<0$ and $-\varsigma_{1}+\frac{240}{119} \varsigma_{2}<0$. So the incipient Hopf bifurcation occurs on the line $\varsigma_{2}=0$ and we obtain a curve $L_{4}:-\varsigma_{1}+\frac{240}{119} \varsigma_{2}=0$.

(4) The secondary Hopf bifurcation solution

$$
y^{2}=\frac{10}{83}\left(12 \varsigma_{2}-\frac{119}{20} \varsigma_{1}\right), r^{2}=-\frac{100}{83}\left(\frac{3}{20} \varsigma_{1}-\varsigma_{2}\right) .
$$

It is easy to see that the secondary Hopf bifurcation solution takes place when $-\varsigma_{1}+\frac{240}{119} \varsigma_{2}<0$ and $-\varsigma_{2}+\frac{3}{20} \varsigma_{1}<0$.

To reveal further the nonlinear dynamic behaviors of the functionally graded materials plate, the waveforms and the phase portraits are depicted. Fig. 2 shows that the trajectory converges to the origin when $\left(\varsigma_{1}, \varsigma_{2}\right)=(0.1,0.2)$. It is also observed from Fig. 3 that trajectory converges to the static bifurcation solution when $\left(\varsigma_{1}, \varsigma_{2}\right)=(-0.2,0.3)$.

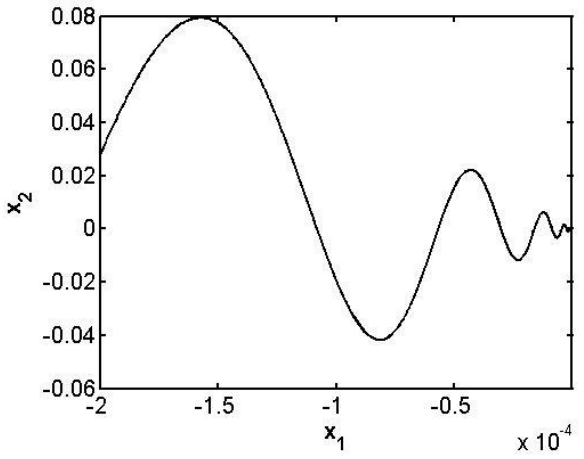

(a)

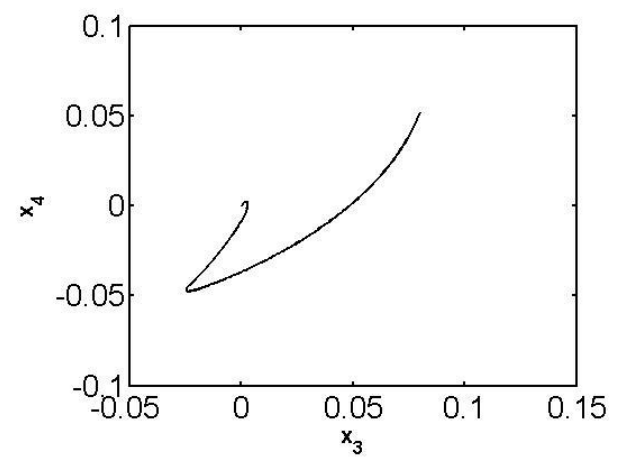

(b)

Figure 2. Trajectory projection starting form initial point $x_{0}=(-0.0002,0.028,0.098,0.032)$ 


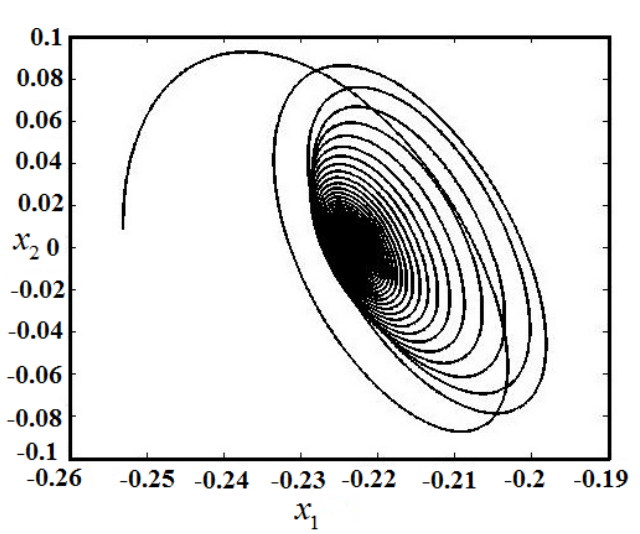

(a)

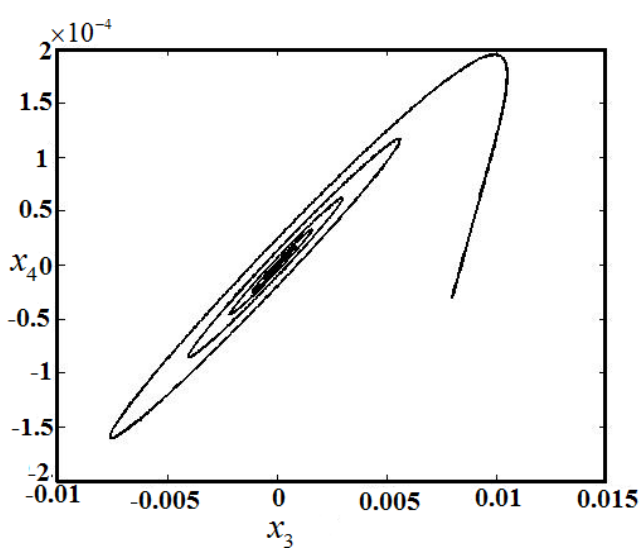

(b)

Figure 3. Trajectory projection starting form initial point $x_{0}=(0.008,-0.05,0.073,0.08)$

\section{Conclusion}

In our paper, we investigate the bifurcation and stability behaviors of the FGMs rectangular plate subjected to transverse and the in-plane excitations. The nonlinear partial differential governing equations of motion are derived by using Hamilton's principle. The nonlinear vibration equations are deduced using Galerkin approach. Based on the normal form theory, the bifurcation theory is utilized to discuss the static bifurcation, incipient Hopf bifurcation and secondary Hopf bifurcation in the vicinity of degenerated equilibrium. The results provide some guidance for the design of the structure of FGMs plate. This technique, based on the method of normal form and bifurcation theory, can be used to deal with general engineering problems.

\section{Acknowledgements}

The authors gratefully acknowledge the support of the National Natural Science Foundations of China (NNSFC) through grant No.11302184, the Scientific Research Fund of Zhejiang Provincial Education Department through grant No. Y201121157 and the Scientific Research Foundation of Xiamen University of Technology through grant No. YKJ13007R.

\section{References}

1. S. Abotula, A. Kidane, V. B. Chalivendra, A. Shukla, Dynamic curving cracks in functionally graded materials under thermo-mechanical loading, Int. J. Solids Struct. 13 (2012) 1637-1655.

2. R. Gunes, J. N. Reddy, Nonlinear analysis of functionally graded circular plates under different loads and boundary conditions, Int. J. Struct. Stab. Dy. 8 (2008) 131-159.

3. D. V. Dung and, L. K. Hoa, Semi-analytical approach for analyzing the nonlinear dynamic torsional buckling of stiffened functionally graded material circular cylindrical shells surrounded by an elastic medium, Appl. Math. Model. 39 (2015) 6951-6967.

4. J. Sladek, V. Sladek, P. Stanak, C. Z. Zhang, M. Wünsche, Analysis of the bending of circular piezoelectric plates with functionally graded material properties by a MLPG method, Eng. Struct. 47 (2013) 81-89.

5. Q. Li, V. P. Lu, K. P. Kou, Three-dimensional vibration analysis of functionally graded material sandwich plates, J. Sound Vib. 311 (2008) 498-515.

6. A. Allahverdizadeh, M. H. Zaei and, M. N. Bahrami, Nonlinear free and forced vibration analysis of thin circular functionally graded plates, J. Sound Vib. 310 (2008) 966-984. 
7. R. Gunes, J. N. Reddy, Nonlinear analysis of functionally graded circular plates under different loads and boundary conditions, Int. J. Struct. Stab. Dy. 8 (2008) 131-159.

8. Y. X. Hao, L. H. Chen, W. Zhang, J. G. Lei, Nonlinear oscillations, bifurcations and chaos of functionally graded materials plate, J. Sound Vib. 312 (2008)862-892.

9. X. H. Zhang, Local bifurcations of nonlinear viscoelastic penel in supersonic flow, Commun Nonlinear Sci Numer Simulat. 18 (2013)1931-1938.

10. C. Anton, J. Deng, Y. S. Wong, Hopf bifurcation analysis of an aeroelastic model using stochastic normal form, J. Sound Vib. 331 (2012)3866-3886.

11. P. Li, Y. R. Yang, H. J. Shi, Hopf and two-multiple semi-stable limit cycle bifurcations of a restrained plate subjected to subsonic flow, J. Sound Vib. 335 (2015) 286-303,.

12. W. Zhang, C. Z. Song, M. Ye, Further studies on nonlinear oscillations and chaos of a rectangular symmetric cross-by laminated plate under parametric excitation, Int. Bifurcat Chaos. 16 (2006) 325-347.

13. Y. X. Hao, W. Zhang, J. Yang, Analysis on nonlinear oscillations of a cantilever FGM rectangular plate based on third-order plate theory and asymptotic perturbation method, Compos Part B: Eng. 42 (2011)402-413. 